\title{
Investigation on Structural, Surface Morphological and Dielectric Properties of Zn-doped $\mathrm{SnO}_{2}$ Nanoparticles
}

\author{
Suresh Sagadevan ${ }^{a *}$ and Jiban Podder ${ }^{b}$ \\ ${ }^{a}$ Department of Physics, AMET University, Chennai 603 112, India \\ ${ }^{b}$ Department of Chemical and Biological Engineering, University of Saskatchewan - USASK, \\ Saskatoon, SK S7N 5A9, Canada
}

Received: October 10, 2015; Accepted: January 11, 2016

\begin{abstract}
Zinc doped Tin oxide $\left(\mathrm{SnO}_{2}\right)$ nanoparticles were prepared by co-precipitation method. The average crystallite size of pure and $\mathrm{Zn}$-doped $\mathrm{SnO}_{2}$ nanoparticles was calculated from the X-ray diffraction (XRD) pattern. The FT-IR spectrum indicated the strong presence of $\mathrm{SnO}_{2}$ nanoparticles. The morphology and the particle size were studied using the scanning electron microscope (SEM) and transmission electron microscope (TEM). The particle size of the $\mathrm{Zn}$-doped $\mathrm{SnO}_{2}$ nanoparticles was also analyzed, using the Dynamic Light Scattering (DLS) experiment. The optical properties were studied by the UV-Visible absorption spectrum. The dielectric properties of $\mathrm{Zn}$-doped $\mathrm{SnO}_{2}$ nanoparticles were studied at different frequencies and temperatures. The ac conductivity of $\mathrm{Zn}$-doped $\mathrm{SnO}_{2}$ nanoparticles was also studied.
\end{abstract}

Keywords: Zn-doped $\mathrm{SnO}_{2}$ nanoparticles, Co-precipitation method, UV-Visible absorption, Dielectric studies

\section{INTRODUCTION}

Tin oxide $\left(\mathrm{SnO}_{2}\right)$, a significant n-type broad direct band gap semiconductor has been the subject of much interest and discussion for researchers because of its numerous and wide ranging applications, such as in flat panel displays, catalysis, heat mirrors, transparent electrodes preparation, gas sensing, etc. ${ }^{1-4}$. Further, recently, this material has created a growing interest as a nanostructured material due to its interesting electrical and optical properties arising out of large surface-to-volume ratio, quantum confinement effect, etc. $^{5-7}$. Owing to high surface-to-volume ratio, the surface atoms play a big role in the properties of nanomaterials, which usually have less adjacent coordinated atoms and can be treated as defects as compared with the bulk atoms. These defects bring on additional electronic states in the band gap, which can mix with the intrinsic states to a substantial extent and which may influence the spacing of the energy levels and the optical properties of nanopowders. A variety of methods were used to prepare $\mathrm{SnO}_{2}$ nanostructures, such as hydrothermal method, polymeric and organometallic precursor synthesis, soncation procedure, microwave synthesis and surfactant-mediated method ${ }^{8-18}$. Zinc is a quite active element. It dissolves in both acids and alkalis. In moist air, however, it reacts to form zinc carbonate. The zinc carbonate forms a thin white crust on the surface which prevents further reaction. Zinc burns in air with a bluish flame. The second largest use of zinc is in making alloys. The mixtures might have properties different from those of the individual metals. In this paper, the preparation of $\mathrm{Zn}$-doped $\mathrm{SnO}_{2}$ nanoparticles and their structural, surface morphology, optical, dielectric and ac conductivity studies were investigated.

\footnotetext{
*e-mail: sureshsagadevan@gmail.com
}

\section{EXPERIMENTAL PROCEDURE}

Co-precipitation method is atomic scale mixing and hence the calcining temperature is required for the formation of final product to lower particle size. Homogeneous mixing of reactant precipitates reduces the reaction temperature. All chemical reagents were commercial with AR purity, and used directly without further purification. Zinc doped $\mathrm{SnO}_{2}$ nanoparticles were prepared by co-precipitation method. The requisite amount of the starting raw materials tin (II) chloride dehydrate and zinc acetate dehydrate were weighed on the percentage of dopant ( $4 \mathrm{~mol} \%$ ) and dissolved into deionized water. The precipitation was achieved by slowly adding aqueous ammonium solution (8M) with the constant stirring until the $\mathrm{pH}$ value reached 10 . The final product was washed several times with deionized water to remove any possible by-products. The filtrate was initially dried at $80^{\circ} \mathrm{C}$ for $12 \mathrm{hrs}$ and calcined around the temperature of $600^{\circ} \mathrm{C}$ for $3 \mathrm{hrs}$ in air atmosphere. The prepared powders were carefully subjected to the following characterization studies. The crystalline size and the structure of the $\mathrm{Zn}$-doped $\mathrm{SnO}_{2}$ nanoparticles was analyzed by X-ray diffraction (XRD) using a powder X-ray diffractometer (Schimadzu model: XRD 6000 with $\mathrm{CuK}_{\alpha}$ radiation and with a diffraction angle between $20^{\circ}$ and $80^{\circ}$. The FTIR spectrum of the $\mathrm{Zn}$-doped $\mathrm{SnO}_{2}$ nanoparticles was obtained using an FTIR model Bruker IFS 66W Spectrometer. The surface morphology of the Zn-doped $\mathrm{SnO}_{2}$ nanoparticles was observed by a scanning electron microscope (SEM) using JEOL; JSM- 67001. Transmission electron microscope (TEM) image was taken using an H-800 TEM (Hitachi, Japan) with an accelerating voltage of $100 \mathrm{kV}$. UV-Visible absorption spectrum for the pure and $\mathrm{Zn}$-doped $\mathrm{SnO}_{2}$ nanoparticles was recorded using a Varian Cary $5 \mathrm{E}$ spectrophotometer in the range of $300-900 \mathrm{~nm}$. The dielectric 
constant, the dielectric loss and the ac conductivity of the pellets of $\mathrm{Zn}$-doped $\mathrm{SnO}_{2}$ nanoparticles in disk form were studied at different temperatures using an HIOKI 3532-50 LCR HITESTER in the frequency range of $50 \mathrm{~Hz}$ to $5 \mathrm{MHz}$.

\section{RESULTS AND DISCUSSION}

\subsection{Structural Analysis}

$\mathrm{X}$-ray powder diffraction (XRD) is a powerful technique used to uniquely identify the crystalline phases present in materials and to measure the structural properties of those phases. X-ray powder diffraction patterns of pure and Zn-doped $\mathrm{SnO}_{2}$ nanoparticles are shown in Fig.1. All the diffraction peaks are well assigned to the tetragonal system of $\mathrm{SnO}_{2}$. It is noteworthy that no diffraction peaks correspond to $\mathrm{Zn}$ oxides and it indicates that increase in the dopant concentration of $\mathrm{Zn}$, causes the peak shift towards the higher angle. The observation of peak broadening indicates the occurrence of smaller crystalline size of $\mathrm{SnO}_{2}$ nanoparticles. As the $\mathrm{Zn}$ content increases, the intensity of XRD peaks decreases and it shows the degradation of crystallinity. This means that $\mathrm{Zn}$ doping in $\mathrm{SnO}_{2}$ produces crystal defects around the dopants and the charge imbalance arising from this defect changes the stoichiometry of the materials. The average nano-crystallite size (D) was calculated using the Scherrer formula (1),

$D=\frac{0.9 \lambda}{\beta \cos \theta}$

where $\lambda$ is the X-ray wavelength, $\theta$ is the Bragg diffraction angle, and $\beta$ is the FWHM of the XRD peak appearing at the diffraction angle $\theta$. The average crystallite sizes of pure and $\mathrm{Zn}$-doped $\mathrm{SnO}_{2}$ nanoparticles were found to be 11 and $14 \mathrm{~nm}$ respectively. The crystalline sizes were estimated from the Scherrer's relation. It indicates that increase in the dopant concentration of $\mathrm{Zn}$, increases the average crystalline size.

\subsection{FTIR Analysis}

Infrared (IR) refers broadly to that part of the electromagnetic spectrum between the visible and microwave regions. FTIR is conceivably the most powerful tool for identifying the functional groups or the types of chemical bonds. FTIR spectrums of pure and $\mathrm{Zn}$-doped $\mathrm{SnO}_{2}$ nanoparticles are shown in Fig.2. It is clearly observed that the peak forms at around $671 \mathrm{~cm}^{-1}$ for pure $\mathrm{SnO}_{2}$. The peak at $671 \mathrm{~cm}^{-1} \mathrm{can}$ be attributed to the stretching vibration of the $\mathrm{O}-\mathrm{Sn}-\mathrm{O}$ bond formed by oxolation reactions. A weak bond at $1628 \mathrm{~cm}^{-1}$ is recognized as the deformation mode of $\mathrm{OH}$ groups. It has also been endorsed that the increase in $\mathrm{Zn}$ content causes the small shift in wave number to lower region.

\subsection{SEM analysis}

Scanning electron microscopy (SEM) is one of the most widely used techniques used in the characterization of nanomaterials and nanostructures. The signals that derive from electron-sample interactions reveal information about the sample including surface morphology of the sample. Fig. 3 shows the SEM micrograph of $\mathrm{Zn}$-doped $\mathrm{SnO}_{2}$ nanoparticles. As shown in Fig.3, the as-synthesized $\mathrm{Zn}$-doped $\mathrm{SnO}_{2}$ nanoparticles consist of fine tiny spherical nanoparticles. The $\mathrm{Zn}$-doped $\mathrm{SnO}_{2}$ nanoparticles appear to be slightly

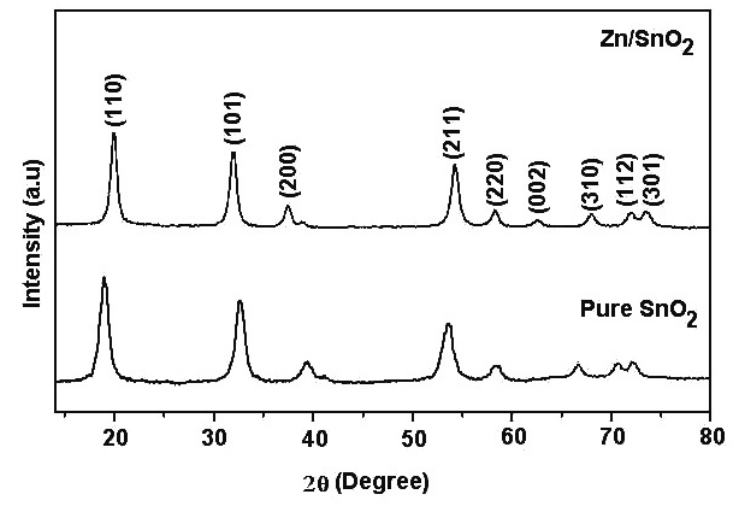

Fig.1 XRD pattern of pure and $\mathrm{Zn}$-doped $\mathrm{SnO} 2$ nanoparticles

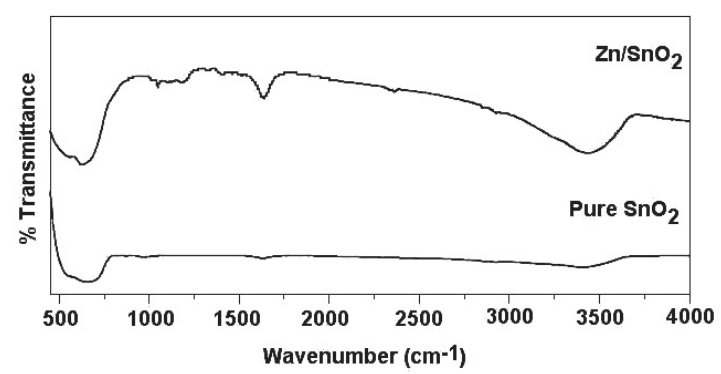

Fig.2. FTIR spectrum of pure and $\mathrm{Zn}$-doped $\mathrm{SnO} 2$ nanoparticles

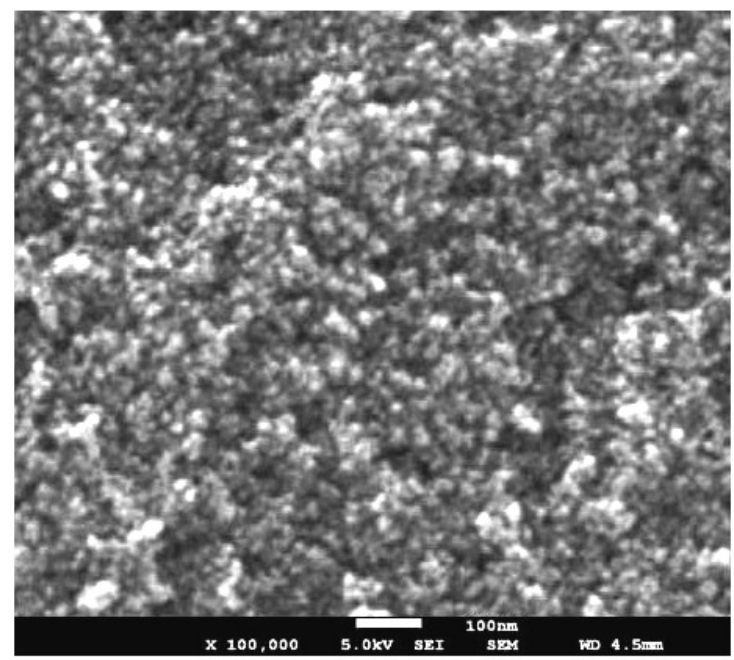

Fig.3 SEM image of $\mathrm{Zn}$-doped $\mathrm{SnO} 2$ nanoparticles

agglomerated spherical ones in the crystallite size range of $10 \sim 12 \mathrm{~nm}$. There are small agglomerated particles and this might be due to the lower calcination temperature.

\subsection{TEM Analysis}

The transmission electron microscope uses a high energy electron beam transmitted through a very thin sample to image and analyze the microstructure of materials with 
atomic scale resolution. The TEM image of $\mathrm{Zn}$-doped $\mathrm{SnO}_{2}$ nanoparticles is shown in Fig.4. TEM micrographs also demonstrate that the formed nanoparticles are homogeneous, with no significant phase separations or coatings on the surface. The particle size-distributions for $\mathrm{Zn}$-doped $\mathrm{SnO}_{2}$ nanoparticles were estimated from TEM images. It is clear from the figures that the grains are segregated together to form large sized agglomerates. It can be seen that the particles are non-uniform in shape and have a particle size distribution in the range of 16-18 $\mathrm{nm}$. Dynamic Light Scattering (DLS) is a very important tool for characterizing the size of nanoparticles in a solution. The DLS of the $\mathrm{Zn}$-doped $\mathrm{SnO}_{2}$ nanoparticles is shown in Fig.5. The dynamic light scattering experiment showed that the particle size of $\mathrm{Zn}$-doped $\mathrm{SnO}_{2}$ nanoparticles was in the range of 10 to $20 \mathrm{~nm}$.

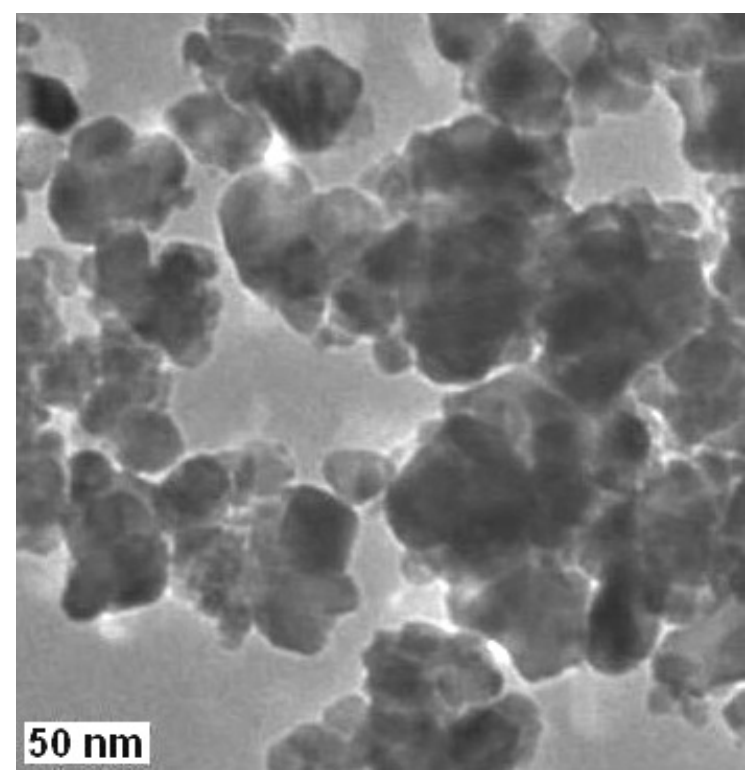

Fig.4.TEM image of $\mathrm{Zn}$-doped $\mathrm{SnO} 2$ nanoparticles

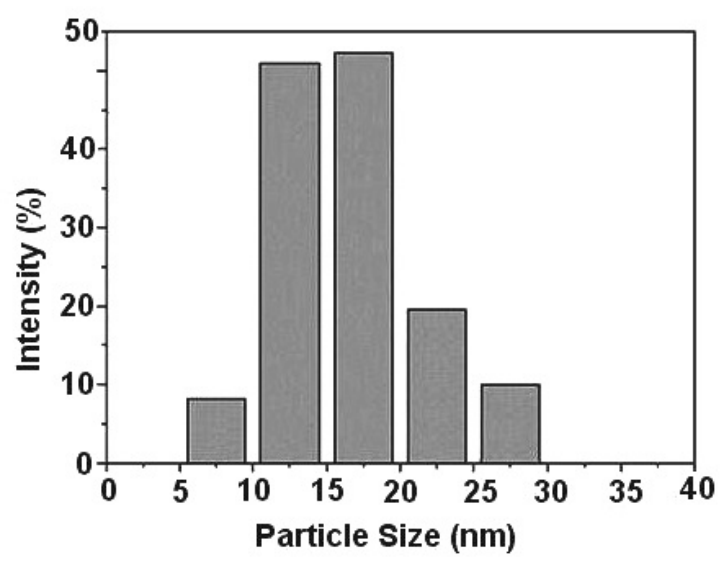

Fig.5. Particle size of $\mathrm{Zn}$-doped $\mathrm{SnO} 2$ nanoparticles

\subsection{Optical Studies}

UV-visible spectroscopy is used when involving the absorption of these high energy lights by atoms or molecules, which cause electronic excitation. The optical properties of pure and $\mathrm{Zn}$-doped $\mathrm{SnO}_{2}$ nanoparticles were characterized by UV-visible absorption spectroscopy. The absorption spectrum of the pure and $\mathrm{Zn}$-doped $\mathrm{SnO}_{2}$ nanoparticles is shown in Fig. 6. It is clearly observed that the absorption edge shifts towards the higher wavelength side (red shift) with the increase in dopant concentration, which agrees well with the reported results ${ }^{19}$. Although a quantum confinement could be expected due to the decrease of the particle size upon doping, a small decrease of the absorption edge is observed corresponding to a red-shift, similar to the one observed in $\mathrm{Co}$ doped $\mathrm{SnO}_{2}$ and Co-doped $\mathrm{ZnO}{ }^{20,21}$. Generally, the wavelength of the maximum excitation absorption decreases as the particle size decreases, as a result of the quantum confinement of the photo generated electron-hole pairs.

The optical absorption coefficient $(\alpha)$ was calculated from transmittance using the following relation

$\alpha=\frac{1}{d} \log \left(\frac{1}{T}\right)$

where $\mathrm{T}$ is the transmittance and $\mathrm{d}$ is the thickness. The study has an absorption coefficient $(\alpha)$ obeying the following relation for high photon energies $(h v)$

$\alpha=\frac{A\left(h v-E_{g}\right)^{1 / 2}}{h v}$

where $\alpha, \mathrm{E}_{g}$ and $A$ are the absorption coefficient, band gap and constant respectively. A plot of variation of $(\alpha h v)^{2}$ versus $h v$ is shown in Fig. 7. The band gap of prepared samples was calculated by extrapolating the rising part of the absorption peak. The estimated band gap values for pure and $\mathrm{Zn}$-doped $\mathrm{SnO}_{2}$ nanoparticles were found to be 3.6 and $3.5 \mathrm{eV}$ respectively. It could be observed that the bandgap value slightly decreased with increase in the dopant concentration of $\mathrm{Zn}$.

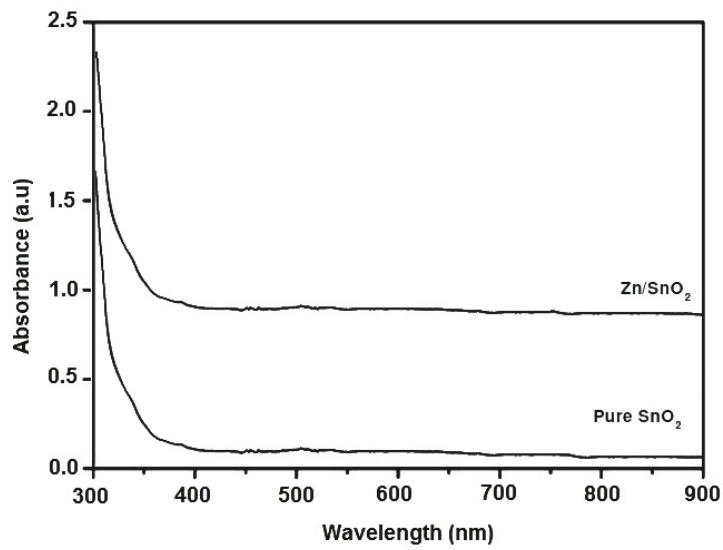

Fig.6. UV-Visible absorption spectrum of pure and $\mathrm{Zn}$-doped $\mathrm{SnO}_{2}$ nanoparticles 


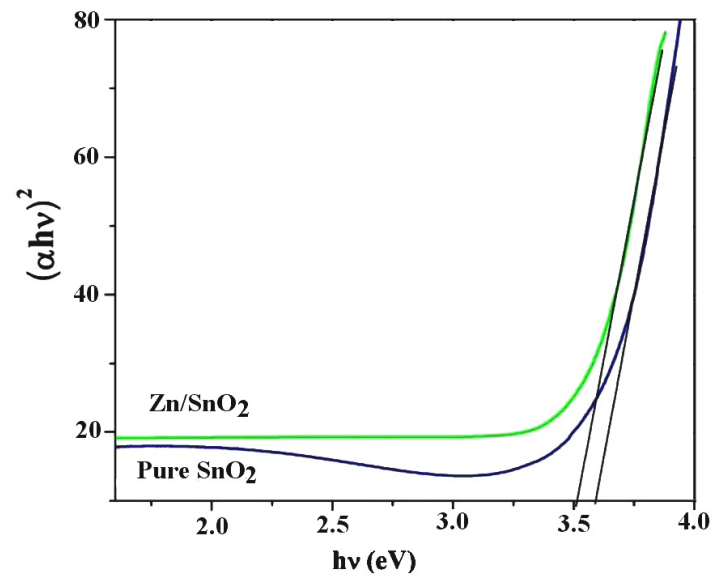

Fig.7 Plot of $(\alpha \mathrm{h} v) 2$ Vs photon energy of pure and $\mathrm{Zn}$-doped $\mathrm{SnO}_{2}$ nanoparticles

\subsection{Dielectric Properties}

The dielectric constant and the dielectric loss of the $\mathrm{Zn}$-doped $\mathrm{SnO}_{2}$ nanoparticles were studied at different temperatures in the frequency region $50 \mathrm{~Hz}-5 \mathrm{MHz}$. The dielectric constant was measured as a function of the frequency at different temperatures as shown in Fig.8, while the corresponding dielectric losses are depicted in Fig.9. The dielectric constant was evaluated using the relation,

$\varepsilon_{r}=\frac{C d}{\varepsilon_{0} A}$

where $\mathrm{d}$ is the thickness of the sample and $\mathrm{A}$ is the area of sample. Fig.8. shows the plot of the dielectric constant $\left(\varepsilon_{\mathrm{r}}\right)$ versus $\log \mathrm{f}$. It can be seen that the dielectric constant decreases with the increase in frequency and becomes almost constant at high frequencies. The polarization decreases with the increase in frequency and then reaches a constant value due to the fact that beyond a certain frequency of external field the hopping between different metal ions cannot follow the alternating field. Due to the application of an electric field, the space charges are moved and dipole moments are created and are called space-charge polarization. In addition to this, these dipole moments are rotated by the field applied resulting in rotation polarization which is also contributing to the high values ${ }^{22}$. Whenever there is an increase in the temperature, more dipoles are created and the value increases ${ }^{23}$. Fig. 9 shows the variation of dielectric loss versus $\log \mathrm{f}$ for various temperatures. It can be observed that the dielectric loss decreases with the increase in the frequency for all temperatures, which may be due to the space charge polarization ${ }^{24}$. It can also be seen that the dielectric loss decreases with increase in the frequency and becomes low at high frequency region, which shows the capability of these materials to be used in high frequency device applications ${ }^{25}$.

\subsection{AC conductivity}

The ac conductivity plot of the pelletized form of $\mathrm{Zn}$-doped $\mathrm{SnO}_{2}$ nanoparticles is shown in Fig.10. It can be observed that the ac conductivity gradually increases with increase

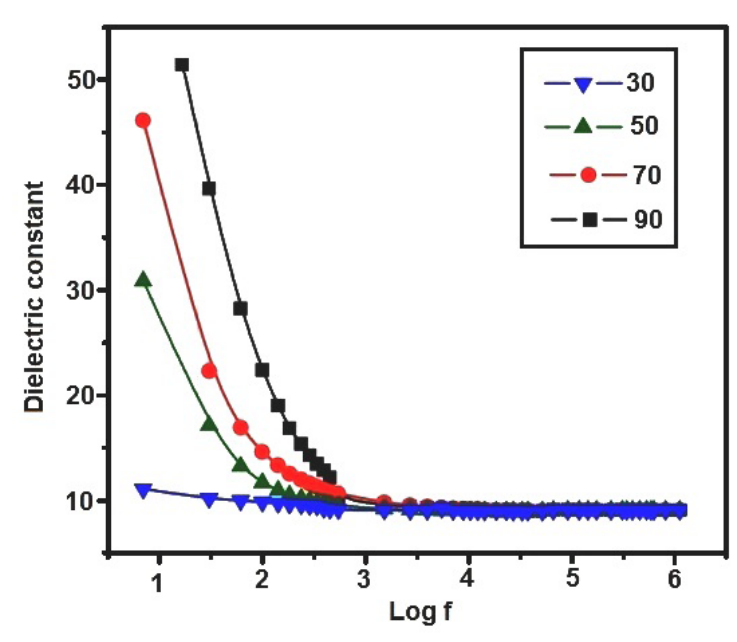

Fig.8. Dielectric constant of $\mathrm{Zn}$-doped $\mathrm{SnO}_{2}$ nanoparticles

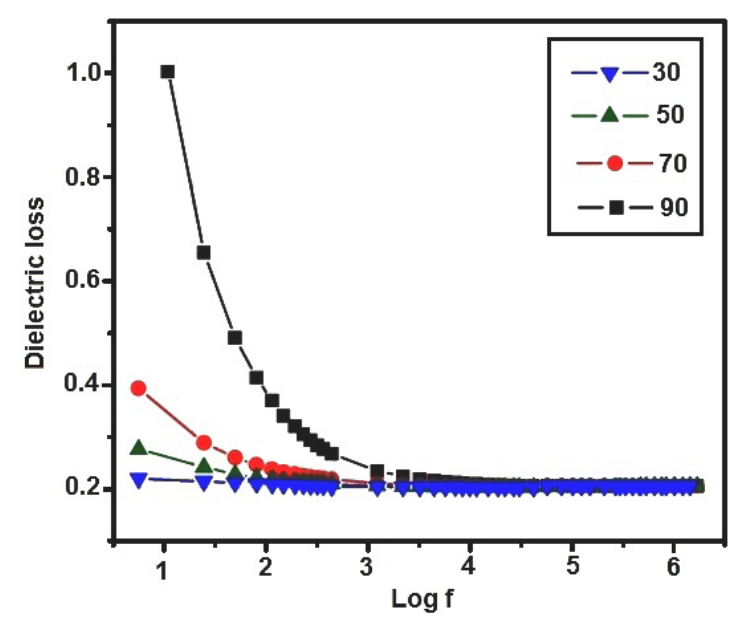

Fig.9. Dielectric loss of $\mathrm{Zn}$-doped $\mathrm{SnO}_{2}$ nanoparticles

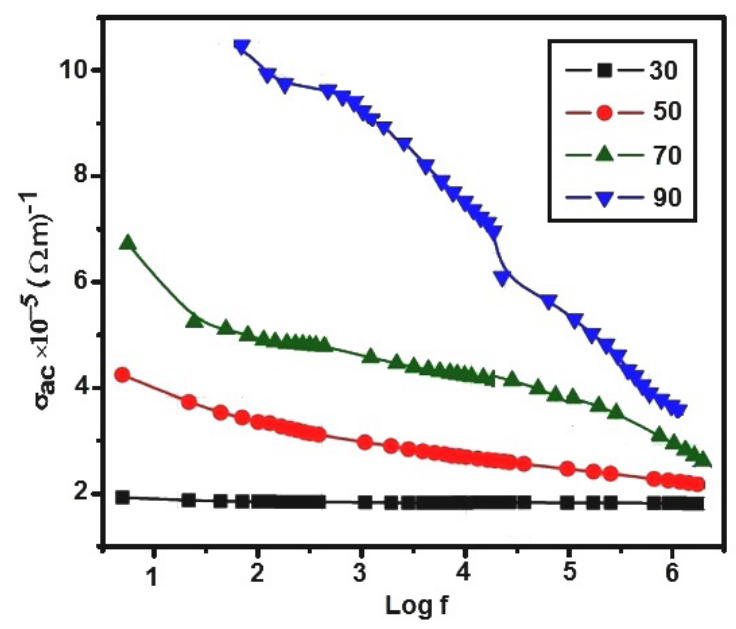

Fig.10. Variation of ac conductivity with frequency at various temperatures 
in the frequency of the applied ac field because the increase in the frequency enhances the electron hopping frequency. It could be observed from the results that the ac conductivity increased with increase in temperature, which showed the semiconducting nature of the sample. Due to the thermionic emission and the tunneling of charge carriers across the barrier, the conductivity increased with the temperature. Because of the small size of the particles, more charge carriers reached the surface of the particles, easily enabling the electron transfer by thermionic emission or tunneling to enhance the conductivity ${ }^{26}$. The ac conductivity of the $\mathrm{Zn}$-doped $\mathrm{SnO}_{2}$ nanoparticles could be calculated by the following relation:

$\sigma_{a c}=2 \pi \varepsilon_{0} \varepsilon_{r} f \tan \delta$

where $\varepsilon_{0}$ is permittivity in free space, $\varepsilon_{\mathrm{r}}$ is dielectric constant, $\mathrm{f}$ is the frequency, and tan $\mathrm{d}$ is the loss factor. There was a small increase in the electrical conductivity of the nanomaterial at the low frequency region for an increase in the frequency and was the same for all temperatures. Conversely, at high frequencies, especially in the $\mathrm{KHz}$ region, there was an abrupt increase in the conductivity and it was enormous at high temperatures which could be attributed to small polaron hopping ${ }^{27}$.

\section{REFERENCES}

1. Zhang Y, Yu K, Li G, Peng D, Zhang Q, Xu F, et al. Synthesis and field emission of patterned $\mathrm{SnO} 2$ nanoflowers. Materials Letters. 2006;6(25):3109-3112

2. Kojima M, Takahashi F, Kinoshita K, Nishibe T, Ichidate M. Transparent furnace made of heat mirror. Thin Solid Films. 2001;392(2): 349-354. doi:10.1016/S0040-6090(01)01056-2

3. Lee JH, Park NG, Shin YJ. Nano-grain SnO2 electrodes for high conversion efficiency SnO2-DSSC. Solar Energy Materials Solar Cells. 2011;95:179-183.

4. Granqvist CG. Transparent conductors as solar energy materials: a panoramic review. Solar Energy Materials Solar Cells. 2007;91:1529-1598.

5. Hulser TP, Wiggers H, Kruis FE, Lorke A. Nanostructured gas sensors and electrical characterization of deposited $\mathrm{SnO} 2$ nanoparticles in ambient gas atmosphere. Sensors Actuators B. 2005;109(1):13-18. doi:10.1016/j.snb.2005.03.012

6. Wang G, Yang Y, Mu Q, Wang Y. Preparation and optical properties of Eu3+-doped tin oxide nanoparticles. Journal of Alloys Compounds. 2010;498(1):81-87. doi:10.1016/j. jallcom.2010.03.107

7. Liu H, Gong S, Hu Y, Zhao J, Liu J, Zheng Z, et al. Thin oxide nanoparticles synthesized by gel combustion and their potential for gas detection. Ceramics International. 2009;35:961-966.

8. Chiu HC, Yeh CS. Hydrothermal synthesis of $\mathrm{SnO} 2$ Nanoparticles and their gas-sensing of alcohol. Journal Physics Chemistry C. 2007;111(20):7256 -7259. DOI: 10.1021/jp0688355

9. Firooz AA, Mahjoub AR, Khodadadi AA. Preparation of $\mathrm{SnO} 2$ nanoparticles and nanorods by using a hydrothermal method at low temperature. Materials Letters. 2008;62:1789-1792.

10. Leite ER, Weber IT, Longo E, Varela JÁ. A new method to control particle size and particle size distribution of $\mathrm{SnO} 2$

\section{CONCLUSIONS}

$\mathrm{Zn}$-doped $\mathrm{SnO}_{2}$ nanoparticles were prepared by co-precipitation method. The formation of $\mathrm{Zn}$-doped $\mathrm{SnO}_{2}$ nanoparticles was confirmed by $\mathrm{X}$-ray diffraction. The average crystallite sizes of pure and $\mathrm{Zn}$-doped $\mathrm{SnO}_{2}$ nanoparticles were found to be 11 and $14 \mathrm{~nm}$. The FT-IR spectrum indicated the strong presence of $\mathrm{SnO}_{2}$ nanoparticles. The SEM revealed the morphology of the synthesized samples and it showed that $\mathrm{Zn}$-doped $\mathrm{SnO}_{2}$ nanoparticles were spherical in shape. The transmission electron microscopic analysis confirmed the prepared $\mathrm{Zn}$-doped $\mathrm{SnO}_{2}$ nanoparticles with the particle size of around $18 \mathrm{~nm}$. The particle size of the $\mathrm{Zn}$-doped $\mathrm{SnO}_{2}$ nanoparticles lying in the range of 10 to $20 \mathrm{~nm}$ was determined using the dynamic light scattering experiment which agreed well with the results of the TEM analysis. Optical properties of the $\mathrm{Zn}$-doped $\mathrm{SnO}_{2}$ nanoparticles were investigated by using UV-Visible absorption spectrum. UV-visible absorption studies showed that the decrease of particle size was accompanied by decrease of the band gap value from $3.6 \mathrm{eV}$ for $\mathrm{SnO}_{2}$ down to $3.5 \mathrm{eV}$ for $\mathrm{Zn}$ doping. The variations of the dielectric constant and the dielectric loss were studied. The dielectric studies revealed that both the dielectric constant and the dielectric loss decreased with an increase in the frequency. The ac conductivity of $\mathrm{Zn}$-doped $\mathrm{SnO}_{2}$ nanoparticles was determined and observed that the particle size is increased with the increase in temperature.

nanoparticles for gas sensor applications. Advanced Materials. 2000;12(13):965-969 .

11. Nayral C, Viala E, Fau P, Senocq F, Jumas JC, Maisonnat A, et al. Synthesis of tin and tin oxide nanoparticles of low size dispersity for application in gas sensing. Chemistry- $A$ European Journal. 2000;6(22):4082-4090.

12. Zhu J, Lu Z, Aruna ST, Aurbach D, Gedanken A. Sonochemical Synthesis of $\mathrm{SnO} 2$ nanoparticles and their preliminary study as Li insertion electrodes. Chemistry of Materials. 2000;12(9):25572566. DOI: $10.1021 / \mathrm{cm} 9906831$

13. Pang G, Chen SG, Koltypin Y, Zaban A, Feng S, Gedanken A. Controlling the particles size of calcined $\mathrm{SnO} 2$ nanocrystals. Nano Letter. 2001;1:723-726.

14. Subramanian V, Burke W, Zhu H, Wei BQ. Novel microwave synthesis of nanocrystalline $\mathrm{SnO} 2$ and Its electrochemical properties. Journal of Physical Chemistry C. 2008;112:45504556.

15. Jouhannaud J, Rossignol J, Stuerga D. Rapid synthesis of tin (IV) oxide nanoparticles by microwave induced thermohydrolysis. Journal of Solid State Chemistry. 2008;181(6):1439-1444.

16. Wang YD, Ma CL, Sun XD, Li HD. Preparation and characterization of $\mathrm{SnO} 2$ nanoparticles with a surfactant-mediated method. Nanotechnology. 2002;13:565-569.

17. Podder J, Roy SS. An investigation of structural and electrical properties of nano Crystalline $\mathrm{SnO} 2$ : $\mathrm{Cu}$ thin films deposited by spray Pyrolysis. Sensors \& Transducers Journal. 2011;134(11):155162.

18. Roy SS, Podder J. Synthesis and optical characterization of pure and $\mathrm{Cu}$ doped $\mathrm{SnO} 2$ thin films deposited by spray pyrolysis. Journal of Optoelectronics and Advanced Materials. 2010;12:1479-1484.

19. Bouaine A, Brihi N, Schmerber G, Ulhaq-Bouillet C, Colis S, Dinia A. Structural, optical and magnetic properties of Co-Doped $\mathrm{SnO} 2$ 
powders synthesized by the coprecipitation technique. Journal of Physical Chemistry C. 2007;111:2924-2928.DOI: 10.1021/ jp066897p

20. Park YR, Kim KJ. Sputtering growth and optical properties of [100]-oriented tetragonal $\mathrm{SnO} 2 \mathrm{SnO} 2$ and its Mn alloy films. Journal of Applied Physics. 2003;94(10):6401-6404.

21. Colis S, Bieber H, Begin-Colin S, Schmerber G, Leuvrey C, Dinia A. Magentic properties of Co-Doped $\mathrm{ZnO}$ diluted magnetic semiconductors prepared by low-temperature mechanosynthesis. Chemical Physics Letters. 2006;422(4-6):529-533. DOI: 10.1016/j.cplett.2006.02.109

22. Suresh S. Synthesis, structural and dielectric properties of zinc sulfide nanoparticles. International Journal of Physical Sciences. 2013;8:1121-1127.
23. Suresh S. Synthesis and electrical properties of $\mathrm{TiO} 2$ nanoparticles using a wet chemical technique. American Journal of Nanoscience and Nanotechnology. 2013;1:27-30.

24. Suresh S. Preparation, structural and electrical properties of tin oxide nanoparticles. Journal of Nanomaterials \& Molecular Nanotechnology. 2015;4:1. doi.org/10.4172/2324-8777.1000157

25. Suresh S. Study of structural, surface morphological and dielectric properties of Cu-Doped tin oxide nanoparticles. Journal of Nano Research. 2015;34:91-97. DOI: 10.4028/www.scientific.net/ JNanoR.34.91

26. Suresh S. Studies on the dielectric properties of CdS nanoparticles. Applied Nanoscience. 2014;4(3):325-329.

27. Suresh S, Arunseshan C. Dielectric Properties of Cadmium Selenide (CdSe) nanoparticles synthesized by solvothermal method. Applied Nanoscience. 2014;4(2):179-184. 